\title{
Computing Criticality of Lines in Power Systems
}

\author{
Ali Pınar \\ Computational Research Div. \\ Lawrence Berkeley National Laboratory \\ Berkeley, CA 94720 \\ Email: apinar@lbl.gov
}

\author{
Adam Reichert \\ Dept. of Computer Science \\ Universityof Illinois \\ Urbana, Illinois, 61801 \\ Email: areiche2@uiuc.edu
}

\author{
Bernard Lesieutre \\ Environmental and Energy Tech. Div. \\ Lawrence Berkeley National Laboratory \\ Berkeley, CA 94720 \\ Email: BCLesieutre@lbl.gov
}

\begin{abstract}
We propose a computationally efficient method based on nonlinear optimization to identify critical lines, failure of which can cause severe blackouts. Our method computes criticality measure for all lines at a time, as opposed to detecting a single vulnerability, providing a global view of the system. This information on criticality of lines can be used to identify multiple contingencies by selectively exploring multiple combinations of broken lines. The effectiveness of our method is demonstrated on the IEEE 30 and 118 bus systems, where we can very quickly detect the most critical lines in the system and identify severe multiple contingencies.
\end{abstract}

\section{INTRODUCTION}

Robust operation of a power network requires identifying unplanned component outages that, if not adequately considered, could lead to costly blackouts. Planning and operating criteria are designed so that "the interconnected power system shall be operated at all times so that general system instability, uncontrolled separation, cascading outages, or voltage collapse, will not occur as a result of any single contingency or multiple contingencies of sufficiently high likelihood" [1]. While the " $N-1$ " criterion is achievableexpend in practice for single contingencies, the " $N-k$ " criteria for multiple contingencies is hard to achieve even for small values of $k$ and moderate system sizes. Exploring multiple contingencies requires new algorithms, since brute-force enumeration is very resource demanding, and often prohibitively expensive. In this work, we propose a mathematical model to measure the criticality of lines in the system, which provides valuable information by itself, and can be used to identify potential multiple contingencies that can cause dramatic blackouts.

Identification of multiple contingencies has recently started to draw a lot of interest. Salmeron, Wood, and Baldick [2] employed a bilevel optimization framework along with mixedinteger programming to analyze the security of the grid under terrorist threat. The critical elements of the grid were identified by maximizing the long term disruption in the system, caused by an attack with limited resources. Arroyo and Galiana used a similar bilevel optimization approach. Donde et al. [3], proposed a method that connected the feasibility boundary of power flow equations with spectral graph theory, when voltages are fixed at their nominal values, and only active power flow constraints are considered. Later, Donde et al. [4] extended their approach to include reactive power and proposed a mixed integer nonlinear programming formulation to identify the most significant blackout that can be caused by a specified number of lines or to identify the minimum of lines to cause a blackout of specified severance. More recently, Lesieutre et al. [5] approached this problem from a graph theoretical perspective, by looking for subgraphs in a given graph that are loosely connected to the rest of the graph and have a significant load/generation mismatch.

Identifying a vulnerability in the a power system is important, however, system robustness requires identifying all vulnerabilities of the system. For instance, a specific broken line can take the system dangerously close to infeasibility, so that any one of a handful of broken lines can cause a significant blackout. In this case the real valuable information is not a pair of lines, but the first line, which is most critical, and the second group of lines any one of which can cause a blackout. Therefore, we need tools that can provide global information on the system, as opposed pointing to specific events, and which can be used to detect not only one but many, and preferably all blackouts. In this work, we define a metric for criticality of each line in a power system, and show how to compute this metric. Such information on criticality of power lines can guide system operators to watch closely those lines that might cause significant disturbance to the system if broken. Moreover, this measure of criticality allows us to selectively investigate different scenarios for multiple contingencies. We would like to stress that this process is different than brute-force enumeration, since we will be investigating a scenario only if we know it is perilous. Therefore, the cases to be examined in detail depends directly on the robustness of the system.

To compute the criticality of a line, we solve the dual problem, in which we compute the expendability of a line. That is, a line is critical if it is not expendable. For this purpose, we introduce line parameters for each line that can limit its functionality, so that a high parameter value means, we are using its functionality close to maximum, and a low parameter value means the line is not really being utilized. To measure the criticality of these lines, we minimize the functionality parameters of all lines constrained by power flow equations. At an optimal solution to this problem, the line parameters represent the core of the system, which is absolutely necessary for the system to stay feasible. At this optimal solution, if a line parameter is high, that means we really need this line for the system to stay intact. Conversely, if a line parameter is low, that means the system can probably 
survive without it. Our experiments on the IEEE 30 and IEEE 118 bus systems show the effectiveness of our method.

\section{PRoblem DESCRIPTION}

\section{A. Computing the criticality of a line}

We consider a lossless power system network having $m$ buses (nodes) and $n$ lines (branches). Let $P$ and $Q$ be, respectively, vectors whose components are given by the active and reactive power injections at the buses. Due to the lossless character of the system, we have $\sum_{i=1}^{m} P_{i}=0$, however, $\sum_{i=1}^{m} Q_{i}>0$ as part of the reactive power is consumed in the network.

We note that reducing the admittance of any transmission line in the system to zero is equivalent to removing the line from service. We thus model line expendability in the system by associating a set of variables $0 \leq \gamma_{i} \leq 1, i=1,2, \ldots, n$, with the line admittances and define the modified admittance of line $i$ as the product of the admittance of line $i$ with $\gamma_{i}$. Then $\gamma_{i}=0$ corresponds to the $i$-th line being removed from service, and thus completely expendable, while $\gamma_{i}=1$, which results in the modified admittance being equal to the nominal admittance, indicates that the line is in service at full capacity.

Representing the network angle variables and voltage magnitudes by vectors $\theta$ and $V$, respectively, and letting $B$ denote a diagonal matrix with the value of the line susceptances on its diagonal ${ }^{1}$, the active and reactive power flow equations (with modified admittances) can be written in matrix form as

$$
\begin{array}{r}
A^{T} E B \Gamma \sin (A \theta)-P=0 \\
-|A|^{T} E B \Gamma \cos (A \theta)+d-Q=0,
\end{array}
$$

where $A$ is the branch-node incidence matrix of the network graph and $|A|_{i, j}=\left|A_{i, j}\right|, E$ is a diagonal matrix with

$$
E_{i, i}=\exp \left((|A| \ln V)_{i}\right), \quad i=1, \ldots, n,
$$

$\Gamma$ is a diagonal matrix with

$$
\Gamma_{i, i}=\gamma_{i}, \quad i=1, \ldots, n,
$$

$d$ is defined by

$$
d_{i}=V_{i}^{2} \times\left(A^{T} B \Gamma A\right)_{i, i}, \quad i=1, \ldots, m,
$$

and $\sin (A \theta)$ denotes a vector whose $i$-th component is equal to $\sin \left((A \theta)_{i}\right)$. Similar notation is used to define $\cos (A \theta)$ and $\ln V$. Refer to [6] for more details on this model.

To measure the criticality of all lines, we try to reduce all line parameters as much as possible, constrained by the feasibility of the power flow equations. In the end, what remains is the core of the system, that is the minimal in the system that is necessary to satisfy the power flow equations. In an optimal solution, if a line parameter $\gamma_{i}$ is close to zero, then this line is not likely to be critical, since we can find a solution to the system while this lines functionality is extremely limited. On the other hand, if a line parameter is

\footnotetext{
${ }^{1}$ It is assumed for simplicity that the lines are lossless and shunt elements are absent. However the mathematical framework and the formulations proposed later do not require this assumption.
}

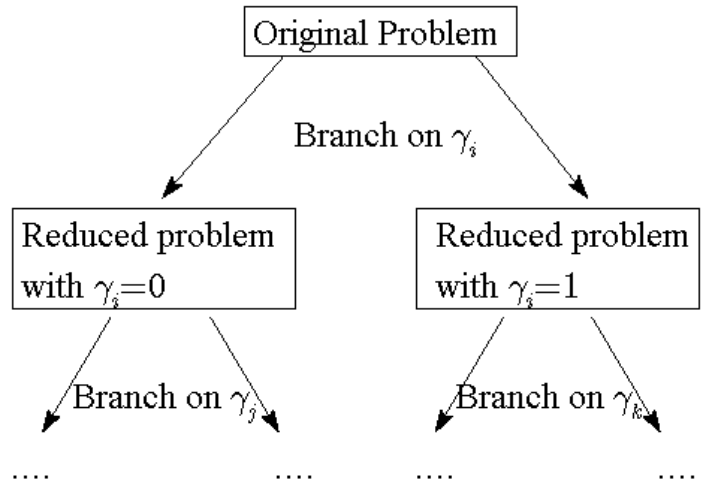

Fig. 1. The branching algorithm

close to 1 , then this line must be critical, since we fail to limit the functionality of this line while keeping the power flow equations feasible.

Mathematically, this problem can be represented as follows.

$$
\begin{array}{ll}
\min _{\gamma, \theta, V} & \|\gamma\| \\
& F(\theta, V, \gamma)=0 \\
& V_{\min } \leq V \leq V_{\max } \\
& -\pi / 2 \leq A \theta \leq \pi / 2 \\
& 0 \leq \gamma \leq 1
\end{array}
$$

where, $F(\theta, V, \gamma)$ corresponds to the power flow equations in (1) and (2). Voltages are bounded with (5). The constraint on angular differences in (6) is required for steady state stability, and the last constraint limits the range of line parameters.

An important point in this formulation is the choice of the norm in the objective function. Since we are trying to identify the most critical lines, we need to favor norms that will minimize the maximum, which excludes the 1-norm. On the other hand, we are trying to find criticality/expendability of all lines at the same time, thus we need a norm that can push each individual line to its minimum, which excludes the $\infty$ norm. In our experiments, we have observed that the 3-norm and the 4-norm has performed best in terms of separating the most critical lines from the others.

\section{B. Identifying Multiple Contingencies}

A solution to the optimization problem in (3)-(7) provides an overall view of the system, and highlights the most critical lines in the system. This information might be used to guide system operators to closely follow certain parts of the system, redispatch the loads to decrease dependence on a certain lines, or develop plans for how to respond to a line failure promptly. This information can also be utilized to identify multiple contingencies, by selectively searching various combinations of failed lines. As discussed before, a complete enumeration or random selections are either prohibitively expensive, or not effective, and the power systems of interest are now too big and complex to rely on engineering intuitions. Therefore, a selective search process is essential. 
Given a solution to the problem in (3)-(7), one can branch on lines by investigating the system when a specified line is already out. This process is illustrated in Fig. 1. In this figure, the left branch corresponds to choosing a line, assuming it is already broken $\left(\gamma_{i}=0\right)$, and investigating the system under this assumption. The right branch corresponds to investigating all scenarios, for which the chosen line is active $\left(\gamma_{i}=1\right)$. Observe that two branches set the line parameter at 0 and 1 , thus explore mutually exclusive combinations. We repeat the process at each branch, to investigate the consequences of different combinations of broken lines. It is worth noting that at each branch, we don't need to stay with the original operating point, but can modify it to reflect how the load might be redispatched after a broken line. In our experiments however, we did not change the initial operating point.

The essence of this technique is how to choose the line to branch on. If we can choose the critical lines, then we can restrict our search process to groups of lines that can potentially have a significant impact when they are concurrently out of service. We use the technique described in the previous section to prioritize the lines to branch on. We first solve the problem in (3)-(7), and choose the line with the highest $\gamma_{i}$ value to branch on. Then at each node we solve the reduced problem, where the value of $\gamma_{i}$ is set to 0 or 1 . This allows us to examine different combinations of broken lines that are likely to cause a significant blackout, without going through an exhaustive search procedure.

A nice feature of the branching algorithm is that it is selfcorrecting, since even if a critical line gets a low criticality value at the first evaluation, this line will still be detected as we explore different combinations and as the critical line missed at the beginning becomes more and more critical.

In our implementation, we maintained a priority queue to examine the most "interesting" cases before others. We are interested in most severe blackouts that are caused by minimum number of lines, and the priority of a scenario in the queue is determined by approximations to these two objectives. Specifically, we assign a priority based on the number of lines already cut, and the $\gamma_{i}$ for the line to branch on.

\section{EXPERIMENTAL RESULTS}

We have applied our proposed techniques to analyze IEEE 30 and IEEE 118 systems [8]. We have used a slightly modified version of the IEEE 30 bus system as in [4], where the generator active power injections are modified so that there is no natural power balance in the system, providing a better test case for vulnerability analysis. This modified data set is presented in detail in [4]. The solutions were computed using the solver SNOPT [7], which uses a sequential quadratic programming algorithm and is designed for nonlinear optimization problems with sparse linear and nonlinear constraints and derivatives.

The first part of our experiments focuses on how we compute the criticality of lines, and the second part focuses

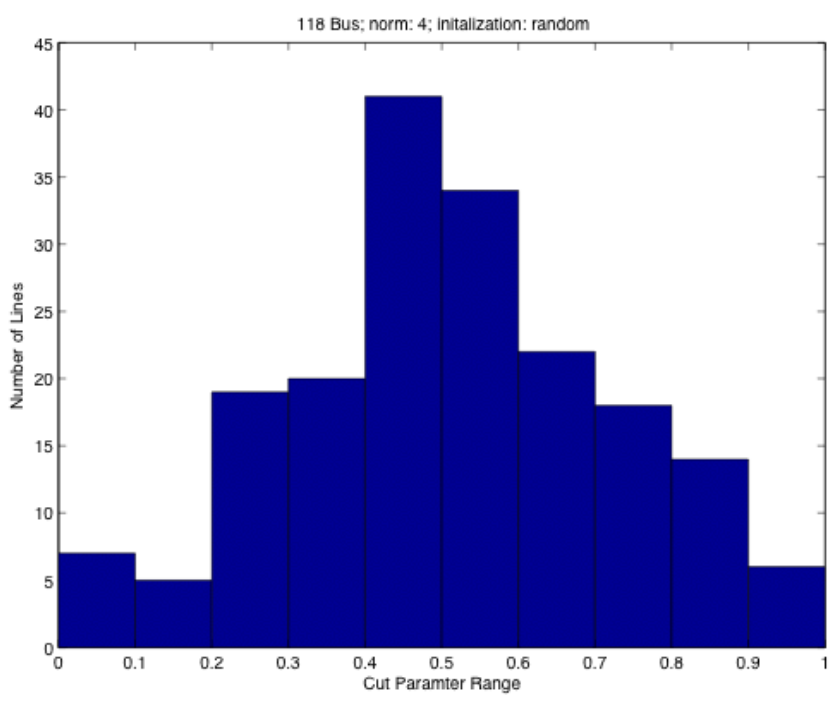

Fig. 2. The histogram of line criticalities

on how we exploit this information to detect multiple contingencies. We evaluate the performance of our method for computing criticality by 4 metrics: consistency (criticalities of lines should be invariant), separation (some lines should be separated from the others as the more critical lines), efficiency (fast solutions are essential for multiple contingency analysis), and accuracy (our measurements should match the real criticalities of lines).

To observe the consistency of our measurements, we solved problem (3)-(7) for the IEEE 118 bus system, starting from 10 different initial solutions using SNOPT, and looked that the deviation from the average. The deviations were consistently $10^{5}$ times less than the computed value, which shows the consistency of the computed results. While the consistency depends on the solver being used, SNOPT is sensitive to the initial starting point, just like other nonlinear solvers, and the consistency of our results show that the posed optimization problem is well-behaved enough to be solved consistently with a state of the art solver.

Our experiments with the IEEE 30 and IEEE 118 bus systems also showed that our technique can identify a small group of lines as the most critical lines. Fig. 2 illustrates the histogram of line criticalities for the IEEE 118 bus system. The lines are put into one of 10 bins accordingly their $\gamma$ values. In the figure, the $\mathrm{x}$-axis show the bins, and the $\mathrm{y}$-axis corresponds to the number of lines in each bin. As seen in this figure, the criticalities follow a bell curve as desired. Only 6 lines have criticality values $>0.9$, which provides a nice separation for the lines that should be investigated first. It is worth noting that this behavior very much depends on the norm being used to measure $\|\gamma\|$. The histogram in the figure was generated with the 4-norm. As discussed in Section II-A, the 1 and $\infty$ norms cannot provide these patterns. In our experiments, the 
2-norm was not very effective either, but we got similar results with the 3 and 5 norms. For different data sets, it might be beneficial to try different, possibly higher norms, if a good separation for $\gamma$ values is not achieved.

We ran our experiments on an Opteron processor with 2.2 $\mathrm{GHz}$ clock speed, and $3 \mathrm{~GB}$ memory per processor. Run-times of our experiments on the IEEE-118 bus system were only 5-6 seconds on average. This makes the proposed branching algorithm for multiple contingency analysis feasible. We want to stress that even with these fast run-times, complete enumeration is not feasible. The complete enumeration for the IEEE-118 system will take around 1 day for $N-2$ security, and 65 days for $N-3$.

Our experiments with the modified 30 bus system has shown the accuracy of our method. At a solution to (3)-(7) the lines with highest $\gamma$ values were lines 16 and 10, which are marked in Figure 3. The criticality of line 16 is obvious, since its removal takes out a generator. However, line 10 is not expected, and its removal causes a significant blackout in the system. Recall that we are working with a modified version of the IEEE-30 bus system, where the generation has been redistributed. We have measured the significance of a blackout as the norm the residual vector when we try to minimize the error for power flow equations. When line 10 was removed the norm of the error was more than $21 \%$ of the norm of the active and reactive power injections, i.e., $\left\|\left(P^{T}, Q^{T}\right)^{T}\right\|$. When we removed lines with low $\gamma$ values, the system was still feasible, or the norm of the error was very small. These results show that our proposed method accurately detects critical lines.

In the next set of experiments, we used the criticality information to find multiple contingencies. The next five lines with highest criticality values were $36,5,8,12$, and 3 . After removing line 36 , and running the optimization algorithm again, we identified, lines $3,5,6$, and 30 as critical lines, and observed that severe blackouts happen when any one of these lines is removed along with line 36 . Similarly, lines 36 or 9 along with line 5; lines $7,9,27,28$, or 38 along with line 8 ; lines 30 or 38 along with line 12 ; lines 28 or 36 along with line 3 , cause severe blackouts. We are currently working on the contingency analysis of the IEEE 118 system. Our experiments showed that while the correlation between criticality values and severity of resulting blackouts is not strict (i.e., a bigger criticality value does not guarantee a more severe blackout), the criticality values provide a nice prioritization for the criticality of lines, and identify the most critical ones.

\section{CONCLUSions AND Future Work}

We propose a nonlinear optimization method to identify critical lines, failure of which can cause severe blackouts. Our method computes a criticality measures for all lines at a time, providing a global view of the system. This information can be used to identify multiple contingencies by selectively exploring multiple combinations of broken lines. The effectiveness of our method is demonstrated on IEEE 30 and 118 bus systems, where we can very quickly detect the most critical lines in the system and identify multiple contingencies.

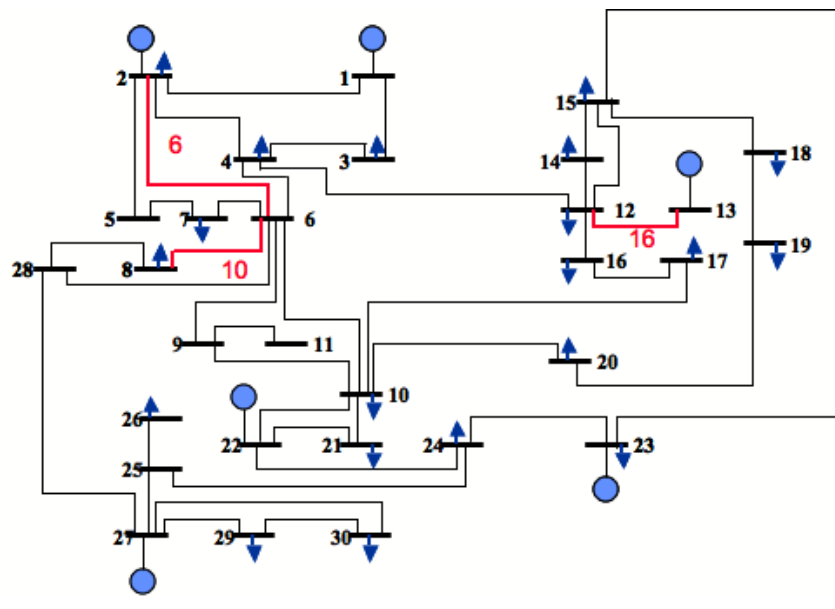

Fig. 3. IEEE 30 bus system

This paper reports our initial results on how to measure criticality of lines in a power system and how to exploit this information, and we plan to extend our work for comprehensive results. We will change blackout severity measurements to incorporate a load shedding model and try several different nonlinear solvers before applying our techniques to continental size problems. While the proposed optimization problem can be solved on a single processor, the number of cases examined can be easily increased by employing multiple processors. We are planning to exploit the inherently parallel nature of the branching algorithm, to use state of the art high performance computing platforms. Our formulations along with the use of high performance computing will allow us to enhance the robustness of the power system.

\section{ACKNOWLEDGMENT}

We would like to thank Vaibhav Donde, Chao Yang, and Juan Meza for fruitful discussions. This work was supported by the Director, Office of Science, Division of Mathematical, Information, and Computational Sciences of U.S. Department of Energy under contract DE-AC03-76SF00098.

\section{REFERENCES}

[1] "Western electricity coordinating council, operating comittee handbook," 2005.

[2] J. Salmeron, K. Wood, and R. Baldick, "Analysis of electric grid security under terrorist threat," IEEE Transactions on Power Systems, vol. 19, pp. 905-912, 2004.

[3] V. Donde, V. Lopez, B. Lesieutre, A. Pinar, C. Yang, and J. Meza, "Identification of severe multiple contingencies in electric power networks," in Proceedings of the 37th North American Power Symposium, Ames, Iowa, 2005.

[4] - "Identification of severe multiple contingencies in electric power systems," IEEE Transactions on Power Systems, submitted.

[5] B. Lesieutre, S. Roy, V. Donde, and A. Pinar, "Power sytem extreme event screening using graph partitioning," in Proceedings of the 38th North American Power Symposium, Carbondale, Illinois, 2006.

[6] E.Scholtz, "Observer-based monitors and distributed wave controllers for electromechanical disturbances in power systems," Ph.D. dissertation, 2004.

[7] "Neos guide: Optimization software." [Online]. Available: http://wwwfp.mcs.anl.gov/otc/Guide/SoftwareGuide/Blurbs/sqopt_snopt.html

[8] "Power system test case archive." [Online]. Available: http://www.ee.washington.edu/research/pstca/ 